\title{
Sweet Corn as Affected by Foliar Application with Amino - and Humic Acids under Different Fertilizer Sources
}

\author{
Entsar E. Ragheb \\ Department of Vegetable Crops, Faculty of Agriculture, \\ Alexandria University, Alexandria, Egypt.
}

\begin{abstract}
7 WO FIELD experiments were conducted at the Experimental Station Farm (at Abies), Faculty of Agriculture, Alexandria University, Egypt, during the two summer seasons of 2014 and 2015. The objective of this investigation was to study the main effects of three different fertilizer sources as well as control $\left(20 \mathrm{~m}^{3} \mathrm{fed}^{-1}\right.$ chicken manure, $30 \mathrm{~m}^{3} \mathrm{fed}^{-1}$ cattle manure and $100-40-60 \mathrm{~kg} \mathrm{NPK} \mathrm{fed}^{-1}$, as recommended rates from each one of the three sources of fertilizer), foliar spray using two sources of growth stimulants as well as the control (spray with distilled water), $\left(2 \mathrm{~g} \mathrm{~L}^{-1}\right.$ amino acid and $2.5 \mathrm{ml} \mathrm{L}^{-1}$ humic acid), and their interaction on growth, yield and its component characters, and some chemical composition of kernels and leaves of sweet corn. The obtained results indicated that the application of chicken manure to sweet corn plants increased the most measured vegetative and kernels quality ( plant height, leaves number plant $^{-1}$, kernels dry matter, reducing sugars, total sugars, T.S.S.), while, the application of either chicken manure or cattle manure, significantly, increased sweet corn yield, ears characteristics and NPK leaves content. Regarding the effect of foliar spray by amino - and humic acids, the results pointed out that the application of amino acid foliar spray were significantly associated with corresponding increases in the most important characters of sweet corn plants. The results concerning of the first order - interaction indicated that the application of chicken - or cattle manure fertilizer combined with the foliar spray with amino - or humic acids, resulted in the highest mean values of the vegetative growth characters, and most of the studied yield and its components characters. The most favorable combination treatment was chicken manure combined with amino acid foliar spray, which gave the highest mean values for the most previous studied characters.
\end{abstract}

Keywords: Sweet corn, Mineral fertilizer, Organic fertilizer, Amino acid and Humic acid.

Sweet corn is one of the most popular vegetables in USA, Canada and Australia. It is become the popular in India and other Asian countries. In Egypt, this untraditional crop has still not getting commercial importance. Moreover, it is an attractive crop for producers to grow because the plant grows quickly and could be harvested as early as $65-90$ days depending on the cultivar type. 
Nevertheless, farmers may get good return by exporting it to many other countries all over the world.

Sweet corn (Zea mays var. rugosa, L., earlier known as Zea mays var. saccharata, Sturt) is an annual grass of the Poaceae family. It also called sugar corn and pole corn is a variety of maize with a high sugar content, since, the grains of sweet corn accumulate two to three times more sugars in the endosperm than the normal starchy maize (Doehlert et al., 1993). Sweet corn is favorable for fresh consumption because of its delicious taste, soft and sugary texture compared to other corn varieties.

Sweet corn is a heavy feeder crop, and proper soil fertility is critical for high yield (Simon and Balabbo, 2015). So, it is requiring high amount of nutrients such as nitrogen, phosphorus, and potassium, which, are usually applied in the form of mineral fertilizers. Moreover, in developing countries, farmers apply high doses of fertilizers and chemical plant protection measures to realize high crop yield. Mineral fertilizer provides readily available nutrients to crops and this is often associated with excessive absorption of nitrate and sulphate that may cause health problems in humans (Noble and Coventry, 2005). Also, adding chemical fertilizers had a harmful effect on the environment (Adediran et al., 2004). Therefore, it is recommended to addition organic matter (plant or animal residues) as a substitute for fertilizer chemistry (Oad, et al., 2004). The addition of organic fertilizers efficiently ensures high production and continuous crops by improving soil properties and increase the development of the roots and activity of microorganisms (Abou El-Magd et al., 2006, Ayoola and Makinde, 2009, Geisseler et al., 2010, Inselsbacher et al., 2010). The beneficial effect of using the different types of organic fertilizer including composting, vermicompost, green manure, animal and chicken manures etc. had been reported to improve growth and yield of sweet corn (Gudugi, et al., 2012, Ghosh et al., 2013, Obi et al., 2013 and Amanolahi-Baharvand et al., 2014) and improve the chemical and biological qualities of the soil which increases crop productivity than chemical fertilizers (Obi \& Ebo, 1995, Boatenget al., 2006, Ghosh, et al., 2013 and Murmur et al., 2013 ).

Recent attention, also, has been given to decreasing pollution sources in modern agriculture. One of the approaches to reduce pollution is the use of biostimulants, which have become commonly used as a safety nature of plant growth regulators, polyamines and vitamins. Amino acids (AA) a well-known bio-stimulant which has positive effects on plant growth, yield and significantly mitigates the injuries caused by a biotic stress (Azimi et al., 2013). Amino acids play multiplier roles in the plant, where, the basic part of the living cells in the plant is protein, which is constituted of amino acids. A need for vital amino acids is known to be the cause of increased efficiency and quality of the products. The studies showed that amino acids have direct or indirect effects on the physiological functions of the plants. Recently, some studies have proved that amino acids are well known as bio-stimulants, which have positive effects on

Egypt. J. Hort. Vol. 43, No. 2 (2016) 
plant growth, yield of many vegetable crops. Saeed et al., (2005) on soybean found that treatments of amino acids significantly improved growth parameters as well as yield and quality. The same results was recorded on strawberry (AboSedera et al., 2010) and garlic (Shalaby and El-Ramady, 2014).

Humic acid (HA) is a promising natural resource that can be used as an alternative to synthetic fertilizers to increase crop production. It exerts either a direct effect, such as on enzymatic activities and membrane permeability, or an indirect effect, mainly by changing the soil structure (Kazemi, 2014). In some studies, humic acid was reported to promote the root length (Cenellar et al., 2002), and to increase the fresh and dry weight of plants (Chen et al., 2004). Due to the positive effect of humic substances on the visible growth of plants, there chemicals have been widely used by the growers.

Keeping in view the above facts, the present study was designed to investigate the effects of different fertilizer sources, growth stimulants and their interactions on growth, yield and quality of sweet corn.

\section{Materials and Methods}

In order to investigate the effects of different fertilizer sources, growth stimulants and their interaction on growth, yield and quality of sweet corn, an experiment was conducted at the Experimental Station Farm, at Abies region, Faculty of Agriculture, Alexandria University, Alexandria, Egypt, through two consecutive summer seasons of 2014 and 2015.

Prior to the initiation of each experiment, soil samples from upper layer of the experimental sites to $20-30 \mathrm{~cm}$ depth were collected and analyzed according to the published procedures of Page et al., (1982). The results of the soil analyses are given in Table 1 . The chemical properties of the used organic sources are presented in Table 2 .

TABLE 1. Some soil physical and chemical properties of the experimental sites, during the two summer seasons of 2014 and 2015.

\begin{tabular}{|c|c|c|c|c|c|c|c|c|c|c|}
\hline \multirow[b]{2}{*}{ Seasons } & \multicolumn{4}{|c|}{ Physical } & \multicolumn{6}{|c|}{ Chemical } \\
\hline & $\begin{array}{c}\text { Sand } \\
(\%)\end{array}$ & $\begin{array}{l}\text { Silt } \\
(\%)\end{array}$ & $\begin{array}{c}\text { Clay } \\
(\%)\end{array}$ & Texture & pH & $\begin{array}{c}\text { EC } \\
\left(\text { ds.m }^{-1}\right)\end{array}$ & $\begin{array}{l}\text { OM } \\
(\%)\end{array}$ & $\begin{array}{c}\mathbf{N} \\
(\mathbf{p p m})\end{array}$ & $\begin{array}{c}\mathbf{P} \\
(\mathbf{p p m})\end{array}$ & $\underset{(\mathbf{p p m})}{\mathbf{K}}$ \\
\hline 2014 & 32.34 & 23.50 & 44.00 & Clay loam & 7.70 & 1.29 & 1.12 & 176.00 & 35.18 & 500.00 \\
\hline 2015 & 31.10 & 22.60 & 45.80 & Clay loam & 8.10 & 1.26 & 2.43 & 163.00 & 32.41 & 459.00 \\
\hline
\end{tabular}


TABLE 2. Chemical analysis of the used chicken and cattle manures.

\begin{tabular}{|c|c|c|c|c|c|c|}
\hline \multirow[b]{2}{*}{ Types of manures } & \multirow[b]{2}{*}{$\begin{array}{c}\text { Organic } \\
\text { carbon } \\
\text { OC }(\%)\end{array}$} & \multirow[b]{2}{*}{$\begin{array}{l}\text { OM } \\
(\%)\end{array}$} & \multirow[b]{2}{*}{$\begin{array}{c}\text { C/N } \\
\text { Ratio }\end{array}$} & \multirow[b]{2}{*}{$\begin{array}{c}N \\
(\%)\end{array}$} & \multicolumn{2}{|c|}{ Available } \\
\hline & & & & & $\begin{array}{c}\mathbf{P} \\
(\mathbf{p p m})\end{array}$ & $\underset{(\mathbf{p p m})}{\mathbf{K}}$ \\
\hline Chicken & 16.58 & 28.59 & 2.50 & 6.62 & 9800 & 1140 \\
\hline Cattle & 6.82 & 11.75 & 6.82 & 1.00 & 6000 & 1500 \\
\hline
\end{tabular}

In both experiments, the used experimental layout was split-plot system in a randomized complete block design (RCBD) with three replications. Each replicate consisted of twelve treatments, which representing the combinations among the two factors, the main factor in this trait is three sources of fertilizer as well as the control (without addition), $\left(20 \mathrm{~m}^{3} \mathrm{fed}^{-1}\right.$ chicken manure, $30 \mathrm{~m}^{3} \mathrm{fed}^{-1}$ cattle manure and $100-40-60 \mathrm{~kg} \mathrm{NPK} \mathrm{fed}^{-1}$, as recommended rates from each one of the three sources of fertilizer). The second factor is foliar spray using two sources of growth stimulants as well as the control (spray with distilled water), ( $1 \mathrm{~g} \mathrm{~L}^{-1}$ amino acid and $2 \mathrm{ml} \mathrm{L}^{-1}$ humic acid).

Regarding the arrangement of the different treatments, different fertilizers sources were stetted as the main plots, foliar spray using growth stimulants were considered as the sub-plots. Each experimental unit area was $11.2 \mathrm{~m}^{2}$, including four rows, four meters long and 0.7 meters width, with plant spacing $25 \mathrm{~cm}$. The same experimental steps were conducted in the first and second seasons of 2014 and 2015 , respectively.

With respect to the two different sources of organic manures, chicken and cattle manures were randomly applied to the surface of each row and incorporated well into about $20-40 \mathrm{~cm}$ depth of the soil one week before sowing process. The forms of three types of mineral fertilizers, NPK were used as follows, ammonium nitrate $(33.5 \% \mathrm{~N})$, calcium superphosphate $\left(15.5 \% \mathrm{P}_{2} \mathrm{O}_{5}\right)$ and potassium sulfate $\left(48.5 \% \mathrm{~K}_{2} \mathrm{O}\right) . \mathrm{N}$ and $\mathrm{K}$ fertilizers were added to the growing plants in three equal parts at 25, 35 and 45 days from planting, whereas, calcium super phosphate was broadcasted throughout the soil preparation (before planting). The foliar application of amino - and humic acid were obtained from solid and liquid commercial products "Amino Strong Plus", 49\% amino acids and $20 \% \mathrm{~N}$ and "Hemo Plus 20", 64\% humic acid, 9\% N, 3\% $\mathrm{P}_{2} \mathrm{O}_{5}$ and 5\% $\mathrm{K}_{2} \mathrm{O}$, respectively. Plants were sprayed with humic acid or amino acid three times at 25 day age, at the beginning of male flowers and in the grain filling stage. The seeds of sweet corn of the hybrid cultivar "Merkur" were sown on the side of the row at 25cm a part on May 15, 2014 and May 25, 2015.

Egypt. J. Hort. Vol. 43, No. 2 (2016) 
In each sub-plot, five plants were randomly chosen from the outer two rows to measure the studied characters as follow, at tasseling and silking stages (after 35 - 40 days from planting time), the vegetative growth characters expressed as plant height $(\mathrm{cm})$, stem diameter $(\mathrm{cm})$, leaves number plant ${ }^{-1}$ and dry matter content (\%) were measured. At the harvesting stage (after 70 days from sowing date, in the milky immature kernels), all husked ears of sweet corn plants in the middle two rows in each experimental unit were harvested to determine the characters, ears yield fed ${ }^{-1}$ and number of ears plant ${ }^{-1}$. The ears yield fed ${ }^{-1}$ was estimated by weighting all harvested husked ears in the middle two rows in each experimental unit and then converted into tonfed ${ }^{-1}$. Five harvested ears from each sub-plot were randomly selected to determine ear length $(\mathrm{cm})$, ear diameter $(\mathrm{cm})$, husked ear weight (g) and un-husked ear weight $(\mathrm{g})$. The kernels were separated by cutting from the cobs of the five selected ears and weighted. Then, the weight of the kernels were divided by the five randomly ears to estimate the kernels weight ear $^{-1}(\mathrm{~g})$. The kernels quality were expressed by the grains constituents from dry matter $(\%)$, reducing sugars $(\%)$, non-reducing sugars (\%), total sugars(\%), starch (\%) and total soluble solids (T.S.S). directly after harvest, samples of ears kernels, were randomly bulked, as previously described from each experimental unit to determine such compositions in grains. Total soluble solids percentages reading were taken on the samples of immature kernels by hand digital refractometer. Total sugars, reducing - and non-reducing sugars and starch were estimated using the procedure of Association of Official Analytical Chemists (A.O.A.C., 1990) and expresses as percentages. The concentrations of $\mathrm{N}, \mathrm{P}$ and $\mathrm{K}$ contents in sweet corn leaves were determined on the basis of dry matter. Nitrogen and phosphorus were determined colorimetrically, using spectrophotometer at 662 and 650 nanometer, according to Evenhuis and Dewaord (1980) and Toth et al., (1948), respectively. Potassium was determined by flam photometer as described by Chapman and Pratt (1961). The determination of the three macro-elements $\mathrm{N}, \mathrm{P}$ and $\mathrm{K}$ were expressed as percentages on dry matter basis.

\section{Statistical analysis}

All obtained data were statistically analyzed using Co-Stat Software (2004), computer program for statistics. Duncan's multiple range test was used to compare the differences among the means of the treatments as elucidated by Steel and Torrie (1980).

\section{Results and Discussions}

\section{Vegetative growth characters}

The results of the main effect of different fertilizer sources and foliar spray by growth stimulants, and their interactions on the vegetative growth, yield and its component characters, kernels quality and chemical composition of leaves of sweet corn plants, in the two summer seasons of 2014 and 2015, are shown in Tables $3,4,5$ and 6 , respectively. 
The results in Table 3 illustrated, generally, that the highest mean values of the three characters plant height, leaves number plant ${ }^{-1}$ and dry matter were given by the application of chicken manure treatment followed by cattle manure and mineral NPK fertilizer, compared with those of the control, respectively, in the two growing seasons. This result in correspondence with that obtained by Materechera and Salagae (2002) who found that addition of both chicken - and cattle manure increase plant height and leaves number plant ${ }^{-1}$ of sweet corn. Also, Efthimiadou et al. (2009) recorded that sweet corn growth was significantly higher with application of organic fertilizer compared with the conventional fertilizer. Increasing the morphological characters of sweet corn after organic manure application may be due to increasing the soil organic matter content, cation exchange capacity and mineral nutrients, which in turn encouraged the plant growth to go forward (Ayoola and Makinde, 2009, Dikinya and Mufwanzala, 2010,Lazcanoet al., 2012 and Jasim et al., 2014). On the other hand, the results showed that the differences among the three sources of fertilizer and control treatment for the character stem diameter, in the two growing seasons, were found to be insignificant.

Table 3 showed also that the application of amino acid foliar spray, gave significantly more number of leaves plant ${ }^{-1}$ and increased plant height and leaves dry matter, compared to the control treatment, in both summer seasons. While, foliar spray by humic acid did not reflect any significant differences with amino acid foliar spray or control treatment for the two characters plant height and leaves number plant ${ }^{-1}$. Previous findings indicated that amino acids can directly or indirectly influence the physiological activities and development of plants (Faten et. al., 2010 and Shalaby and El-Ramady, 2014), moreover, foliar application of amino effectively increased plant height and number of leaves of garlic, Shalay and El-Ramady (2014). Foliar spray with humic acid affected positively on vegetative growth of tomato, onion and soybean crops, which were recorded by Kazemi (2014), Kandil et al., (2013) and Saeed et al. (2005), respectively. Concerning the character stem diameter, the data showed that the differences among the mean values were not high enough to be significant, in the two growing seasons.

Data in Table 3 illustrated that the interaction between fertilization sources and foliar spray by growth stimulants, significantly affected on the mean values of leaves number plant ${ }^{-1}$ and leaves dry matter and significantly increased the plant height. While, the mean values of stem diameter character did not differ significantly by the interaction effect between the two studied factors, during the two seasons. The optimum interactive treatment combination for the four studied characters was chicken manure fertilizer plus the foliar spray with amino - or humic acid, in both growing seasons. 
TABLE 3. Effect of fertilizer sources, growth stimulants and their interactions on vegetative growth characters of sweet corn, during the seasons of 2014 and 2015.

\begin{tabular}{|c|c|c|c|c|c|c|c|c|c|}
\hline \multirow{2}{*}{\multicolumn{2}{|c|}{ Treatments }} & \multicolumn{4}{|c|}{2014} & \multicolumn{4}{|c|}{2015} \\
\hline & & $\begin{array}{c}\text { Plant height } \\
\text { (cm) }\end{array}$ & $\begin{array}{c}\text { Stem } \\
\text { diameter } \\
(\mathrm{cm})\end{array}$ & \begin{tabular}{|c|} 
Leaves \\
No. plant \\
\end{tabular} & $\begin{array}{c}\text { Leaves } \\
\text { dry } \\
\text { mater } \\
(\%)\end{array}$ & \begin{tabular}{|c|} 
Plant \\
height $(\mathrm{cm})$
\end{tabular} & $\begin{array}{c}\text { Stem } \\
\text { diameter } \\
(\mathrm{cm})\end{array}$ & \begin{tabular}{|c|} 
Leaves \\
No. plant \\
\end{tabular} & $\begin{array}{c}\text { Leaves } \\
\text { dry mater } \\
(\%)\end{array}$ \\
\hline \multicolumn{10}{|c|}{ Fertilizer sources } \\
\hline \multicolumn{2}{|l|}{ Control } & $111.54 \mathrm{~b}$ & $2.38 \mathrm{a}$ & $7.49 \mathrm{~b}$ & $27.49 \mathrm{~d}$ & $116.58 \mathrm{~b}$ & $2.66 \mathrm{a}$ & $8.44 \mathrm{~b}$ & $30.04 \mathrm{c}$ \\
\hline \multicolumn{2}{|c|}{ Mineral fertilizer } & $135.27 \mathrm{a}$ & $2.58 \mathrm{a}$ & $8.41 \mathrm{a}$ & $36.18 \mathrm{c}$ & $135.99 \mathrm{a}$ & $2.88 \mathrm{a}$ & $9.43 \mathrm{a}$ & $37.75 \mathrm{~b}$ \\
\hline \multicolumn{2}{|c|}{ Cattle manure } & $131.33 \mathrm{a}$ & $2.58 \mathrm{a}$ & $8.09 \mathrm{ab}$ & $44.12 \mathrm{~b}$ & $130.16 \mathrm{a}$ & $2.93 \mathrm{a}$ & $9.15 \mathrm{a}$ & $46.74 \mathrm{a}$ \\
\hline \multicolumn{2}{|c|}{ Chicken manure } & $142.22 \mathrm{a}$ & $2.63 \mathrm{a}$ & $8.55 \mathrm{a}$ & $51.11 \mathrm{a}$ & $147.30 \mathrm{a}$ & $3.19 \mathrm{a}$ & $9.69 \mathrm{a}$ & $53.05 \mathrm{a}$ \\
\hline \multicolumn{10}{|c|}{ Growth stimulants } \\
\hline \multicolumn{2}{|l|}{ Control } & $134.83 \mathrm{~b}$ & $2.51 \mathrm{a}$ & $7.68 \mathrm{~b}$ & $37.09 \mathrm{~b}$ & $129.50 \mathrm{~b}$ & $2.79 \mathrm{a}$ & $8.72 \mathrm{~b}$ & $37.34 \mathrm{~b}$ \\
\hline \multicolumn{2}{|c|}{ Amino acid } & $135.61 \mathrm{a}$ & $2.56 \mathrm{a}$ & $8.61 \mathrm{a}$ & $42.40 \mathrm{a}$ & $140.56 \mathrm{a}$ & $2.98 \mathrm{a}$ & $9.65 \mathrm{a}$ & $44.34 \mathrm{a}$ \\
\hline \multicolumn{2}{|c|}{ Humic acid } & $129.83 \mathrm{ab}$ & $2.55 \mathrm{a}$ & $8.12 \mathrm{ab}$ & $39.67 \mathrm{~b}$ & $134.95 \mathrm{ab}$ & $2.98 \mathrm{a}$ & $9.17 \mathrm{ab}$ & $44.01 \mathrm{a}$ \\
\hline \multicolumn{10}{|c|}{ Fertilizer sources x Growth stimulants } \\
\hline \multirow{3}{*}{ Control } & Control & $102.83 \mathrm{f}$ & $2.33 \mathrm{a}$ & $7.16 \mathrm{~d}$ & $24.24 \mathrm{~g}$ & $107.93 \mathrm{e}$ & $2.58 \mathrm{~b}$ & $7.41 \mathrm{~d}$ & $25.65 \mathrm{f}$ \\
\hline & Amino & $120.96 \mathrm{de}$ & $2.41 \mathrm{a}$ & $7.50 \mathrm{~b}-\mathrm{d}$ & $29.85 \mathrm{f}$ & $125.98 \mathrm{~cd}$ & $2.71 \mathrm{ab}$ & $8.60 \mathrm{~cd}$ & $32.06 \mathrm{ef}$ \\
\hline & Humic & $110.83 \mathrm{ef}$ & $2.33 \mathrm{a}$ & $7.33 \mathrm{~cd}$ & $28.38 \mathrm{fg}$ & $115.83 \mathrm{de}$ & $2.63 \mathrm{ab}$ & $8.20 \mathrm{~d}$ & $32.06 \mathrm{ef}$ \\
\hline \multirow{3}{*}{ Mineral } & Control & $123.33 \mathrm{c}-\mathrm{e}$ & $2.41 \mathrm{a}$ & $7.91 \mathrm{a}-\mathrm{d}$ & 32.38 ef & $126.99 \mathrm{~cd}$ & $2.71 \mathrm{ab}$ & $8.96 \mathrm{~b}-\mathrm{d}$ & $32.43 \mathrm{~d}-\mathrm{f}$ \\
\hline & Amino & $132.33 \mathrm{~b}-\mathrm{d}$ & $2.45 \mathrm{a}$ & $7.99 \mathrm{a}-\mathrm{d}$ & $40.54 \mathrm{~cd}$ & $137.28 \mathrm{bc}$ & $2.83 \mathrm{ab}$ & $9.16 \mathrm{a}-\mathrm{d}$ & $40.61 \mathrm{~cd}$ \\
\hline & Humic & $132.33 \mathrm{~b}-\mathrm{d}$ & $2.41 \mathrm{a}$ & $7.99 \mathrm{a}-\mathrm{d}$ & $35.63 \mathrm{de}$ & $137.26 \mathrm{bc}$ & $2.71 \mathrm{ab}$ & 8.99 a-d & $39.70 \mathrm{c}-\mathrm{e}$ \\
\hline \multirow{3}{*}{ Cattle } & Control & $132.83 \mathrm{~b}-\mathrm{d}$ & $2.58 \mathrm{a}$ & $8.16 \mathrm{a}-\mathrm{d}$ & $41.40 \mathrm{c}$ & $137.92 \mathrm{bc}$ & $2.88 \mathrm{ab}$ & $9.17 \mathrm{a}-\mathrm{d}$ & $41.51 \mathrm{bc}$ \\
\hline & Amino & $138.33 \mathrm{ab}$ & $2.74 \mathrm{a}$ & 8.56.ab & $47.40 \mathrm{ab}$ & $143.72 \mathrm{ab}$ & $3.04 \mathrm{ab}$ & $9.68 \mathrm{a}-\mathrm{c}$ & $50.28 \mathrm{a}$ \\
\hline & Humic & $134.33 \mathrm{bc}$ & $2.58 \mathrm{a}$ & $8.50 \mathrm{a}-\mathrm{c}$ & $43.56 \mathrm{bc}$ & $139.15 \mathrm{~b}$ & $3.04 \mathrm{ab}$ & $9.50 \mathrm{a}-\mathrm{c}$ & $49.35 \mathrm{ab}$ \\
\hline \multirow{3}{*}{ Chicken } & Control & $139.16 \mathrm{ab}$ & $2.74 \mathrm{a}$ & $8.66 \mathrm{ab}$ & $50.35 \mathrm{a}$ & $144.07 \mathrm{ab}$ & $3.13 \mathrm{ab}$ & $9.81 \mathrm{ab}$ & $51.04 \mathrm{a}$ \\
\hline & Amino & $149.99 \mathrm{a}$ & $2.83 \mathrm{a}$ & $8.99 \mathrm{a}$ & $51.81 \mathrm{a}$ & $154.95 \mathrm{a}$ & $3.55 \mathrm{a}$ & $10.11 \mathrm{a}$ & $54.08 \mathrm{a}$ \\
\hline & Humic & $145.83 \mathrm{ab}$ & $2.75 \mathrm{a}$ & $8.83 \mathrm{a}$ & $51.16 \mathrm{a}$ & $149.02 \mathrm{ab}$ & $3.21 \mathrm{ab}$ & $9.83 \mathrm{ab}$ & $54.02 \mathrm{a}$ \\
\hline
\end{tabular}

Yield and its components

Pertaining to the results of the effect of the sources of fertilization on yield and ear characteristics, Table 4 illustrated, generally, that the application of either chicken manure or cattle manure, significantly, increased the ear length, ear diameter, husked ear weight, un-husked ear weight, kernel weight ear ${ }^{-1}$, ears number plant ${ }^{-1}$ and ears yield fed ${ }^{-1}$ compared with those of the control, in the two summer seasons. Also, using NPK mineral fertilizer increased significantly the previous means characters, compared with the control, but, still less than the two sources of organic manure. This may be due to that organic fertilizers can increase microbial activity in soils between $16 \%$ and $20 \%$ as compared to inorganic fertilizers (Dinesh et al., 2010), and, also, organic manure can offer enough nitrogen to micro-organisms that are living in the soil, which convert the nutrients from unavailable to available form for plants, during the growing period, which was reflected on ear characters and yield of sweet corn. Application by organic fertilizer was superior in influencing yield of sweet corn and its components as mentioned by Efthimiadon et al. (2009), Lazcano et al. (2011), Gudugi et al. (2012) and Jasim et al. (2014).

Concerning the effects of foliar spray by amino- or humic acid on ear characteristics and ears yield fed $^{-1}$, data in Table 4 showed that amino acid foliar spray significantly increased these characters, compared with the both

Egypt. J. Hort. Vol. 43, No. 2 (2016) 
treatments, humic acid foliar spray and control, with only two exceptions. These exceptions were noticed on mean values of ear length and ear diameter characters, which did not differ significantly from mean values of foliar spray by humic acid treatment, during the two growing seasons. The later results can support the finding of (Ebrahimi et al., 2014), who reported that amino acid foliar application increased ears number plant $^{-1}$ and yield of sweet corn. Similar results on the stimulatory effects of amino acid on other plants were noticed by Shalaby and El-Ramady (2014), who found that foliar application of amino acid showed the heaviest garlic bulb weight.

It is clear from data in Table 4 that the interaction effects between each of the fertilizer sources and foliar application by growth stimulants on ear characteristics and ear yield fed $^{-1}$ were significant, but, with different magnitudes, in both years. Application of chicken manure combined with of amino acid gave significantly the highest mean values of these characters, followed by the application of chicken manure with foliar spray by humic acid with insignificant differences between one another, in both seasons.

TABLE 4. Effect of fertilizer sources, growth stimulants and their interactions on ears yield and its components of sweet corn, during the seasons of 2014 and 2015.

\begin{tabular}{|c|c|c|c|c|c|c|c|c|c|c|c|c|c|c|c|}
\hline \multirow{2}{*}{\multicolumn{2}{|c|}{ Seasons }} & \multicolumn{6}{|c|}{2014} & & \multicolumn{7}{|c|}{2015} \\
\hline & & $\begin{array}{c}\text { Ear } \\
\text { length } \\
(\mathrm{cm})\end{array}$ & \begin{tabular}{|c|} 
Ear \\
diamete \\
$\mathbf{r}(\mathbf{c m})$
\end{tabular} & $\begin{array}{c}\text { Husked } \\
\text { ear } \\
\text { weight (g) }\end{array}$ & \begin{tabular}{|c|}
$\begin{array}{c}\text { Un- } \\
\text { husked }\end{array}$ \\
ear \\
weight \\
(g)
\end{tabular} & $\begin{array}{c}\text { Kernels } \\
\text { weight } \\
\text { ear }^{-1}(\mathrm{~g})\end{array}$ & $\begin{array}{c}\text { Ears } \\
\text { number } \\
\text { plant }^{-1}\end{array}$ & $\begin{array}{c}\text { Ears } \\
\text { yield } \\
{\text { ton } \text { fed }^{-1}}^{-1}\end{array}$ & $\begin{array}{c}\text { Ear } \\
\text { length } \\
(\mathrm{cm})\end{array}$ & $\begin{array}{c}\begin{array}{c}\text { Ear } \\
\text { diameter } \\
(\mathbf{c m})\end{array} \\
\end{array}$ & $\mid \begin{array}{c}\text { Husked } \\
\text { ear } \\
\text { weight } \\
(\mathrm{g})\end{array}$ & $\begin{array}{c}\text { Un- } \\
\text { husked } \\
\text { ear } \\
\text { weight (g) }\end{array}$ & $\begin{array}{c}\text { Kernels } \\
\text { weight } \\
\text { ear }^{-1}(g)\end{array}$ & \begin{tabular}{|c} 
Ears \\
numbe \\
r plant \\
1
\end{tabular} & $\begin{array}{c}\text { Ears } \\
\text { yield } \\
\text { ton fed } \\
1\end{array}$ \\
\hline \multicolumn{16}{|c|}{ Fertilizer sources } \\
\hline \multicolumn{2}{|c|}{ Control } & $18.23 \mathrm{c}$ & $3.97 \mathrm{c}$ & $135.00 \mathrm{c}$ & $91.00 \mathrm{~b}$ & $9.16 \mathrm{~b}$ & $1.30 \mathrm{~d}$ & $4.82 \mathrm{c}$ & $18.07 \mathrm{c}$ & $3.92 \mathrm{c}$ & $160.00 \mathrm{~b}$ & $0 \mathrm{c}$ & $6 \mathrm{~b}$ & $1.46 \mathrm{c}$ & $5.00 \mathrm{c}$ \\
\hline \multicolumn{2}{|c|}{$\begin{array}{l}\text { Mineral } \\
\text { fertilizer }\end{array}$} & $19.80 \mathrm{~b}$ & $4.72 \mathrm{~b}$ & $236.50 \mathrm{~b}$ & $194.66 \mathrm{a}$ & $166.33 \mathrm{a}$ & $1.57 \mathrm{c}$ & $6.53 \mathrm{~b}$ & $19.68 \mathrm{~b}$ & $4.96 \mathrm{~b}$ & $251.50 \mathrm{a}$ & $219.66 \mathrm{~b}$ & $186.33 \mathrm{a}$ & $1.83 \mathrm{~b}$ & $7.53 \mathrm{~b}$ \\
\hline \multicolumn{2}{|c|}{ Cattle manure } & $20.06 \mathrm{~b}$ & $4.90 \mathrm{a}$ & $244.66 \mathrm{ab}$ & $200.83 \mathrm{a}$ & $172.50 \mathrm{a}$ & $1.62 \mathrm{~b}$ & $6.86 \mathrm{ab}$ & $19.90 \mathrm{ab}$ & $5.12 \mathrm{a}$ & $256.50 \mathrm{a}$ & $225.83 \mathrm{ab}$ & $186.33 \mathrm{a}$ & $1.84 \mathrm{~b}$ & $7.87 \mathrm{~b}$ \\
\hline \multicolumn{2}{|c|}{ Chicken manure } & $21.04 \mathrm{a}$ & $4.93 \mathrm{a}$ & $278.00 \mathrm{a}$ & $210.83 \mathrm{a}$ & $190.83 \mathrm{a}$ & $1.79 \mathrm{a}$ & $7.74 \mathrm{a}$ & $20.84 \mathrm{a}$ & $5.19 \mathrm{a}$ & $291.16 \mathrm{a}$ & $249.16 \mathrm{a}$ & $210.00 \mathrm{a}$ & $2.03 \mathrm{a}$ & $8.50 \mathrm{a}$ \\
\hline \multicolumn{16}{|c|}{ Growth stimulants } \\
\hline \multicolumn{2}{|c|}{ Control } & $18.86 \mathrm{~b}$ & $4.49 \mathrm{~b}$ & $213.00 \mathrm{c}$ & $168.75 \mathrm{~b}$ & $142.50 \mathrm{~b}$ & $1.71 \mathrm{~b}$ & $5.76 \mathrm{c}$ & $18.73 \mathrm{~b}$ & $3.63 \mathrm{a}$ & $238.00 \mathrm{~b}$ & $193.12 b$ & $162.50 \mathrm{~b}$ & $1.71 \mathrm{~b}$ & $6.14 \mathrm{c}$ \\
\hline \multicolumn{2}{|c|}{ Amino acid } & $20.51 \mathrm{a}$ & $4.73 \mathrm{a}$ & $235.37 \mathrm{a}$ & $185.12 \mathrm{a}$ & $161.75 \mathrm{a}$ & $1.92 \mathrm{a}$ & $7.50 \mathrm{a}$ & $20.31 \mathrm{a}$ & $3.97 \mathrm{a}$ & $253.62 \mathrm{a}$ & $213.25 \mathrm{a}$ & $181.75 \mathrm{a}$ & $1.92 \mathrm{a}$ & $7.90 \mathrm{a}$ \\
\hline \multicolumn{2}{|c|}{ Humic acid } & $19.86 \mathrm{a}$ & $4.67 \mathrm{ab}$ & $222.25 \mathrm{~b}$ & $169.12 \mathrm{~b}$ & $144.87 \mathrm{~b}$ & $1.74 \mathrm{~b}$ & $6.93 \mathrm{~b}$ & $19.83 \mathrm{a}$ & $3.79 \mathrm{a}$ & $241.37 \mathrm{~b}$ & $201.62 \mathrm{~b}$ & $64.87 \mathrm{~b}$ & $1.74 \mathrm{~b}$ & $6.89 \mathrm{~b}$ \\
\hline \multicolumn{16}{|c|}{ Fertilizer sources $\mathrm{x}$ Growth stimulants } \\
\hline \multirow{3}{*}{ Control } & ontrol & $17.83 \mathrm{e}$ & $3.80 \mathrm{~d}$ & $116.50 \mathrm{f}$ & $.50 \mathrm{f}$ & $56.50 \mathrm{f}$ & $1.16 \mathrm{e}$ & $3.59 \mathrm{e}$ & $17.60 \mathrm{e}$ & $3.07 \mathrm{e}$ & $141.50 \mathrm{e}$ & $96.50 \mathrm{f}$ & $6.50 \mathrm{f}$ & $1.16 \mathrm{e}$ & $3.09 \mathrm{~g}$ \\
\hline & Amino & $17.86 \mathrm{e}$ & $4.08 \mathrm{~d}$ & $148.50 \mathrm{e}$ & $104.00 \mathrm{e}$ & $78.50 \mathrm{e}$ & $1.67 \mathrm{~cd}$ & $4.30 \mathrm{de}$ & $18.75 \mathrm{de}$ & $4.37 \mathrm{~cd}$ & $173.50 \mathrm{~d}$ & $124.00 \mathrm{e}$ & $98.50 \mathrm{e}$ & $1.67 \mathrm{~cd}$ & $4.80 \mathrm{f}$ \\
\hline & Humic & $18.83 \mathrm{de}$ & $4.04 \mathrm{~d}$ & $140.00 \mathrm{e}$ & $97.50 \mathrm{e}$ & 72.50ef & $1.55 \mathrm{~d}$ & $3.60 \mathrm{e}$ & $17.80 \mathrm{e}$ & $4.3 \mathrm{~d}$ & $165.00 \mathrm{de}$ & $122.50 \mathrm{e}$ & $92.50 \mathrm{ef}$ & $1.55 \mathrm{~d}$ & $4.11 \mathrm{f}$ \\
\hline \multirow{3}{*}{ Mineral } & Control & 19.00de & $4.55 \mathrm{c}$ & $233.00 \mathrm{~d}$ & $185.00 \mathrm{~d}$ & $160.00 \mathrm{~d}$ & $1.71 \mathrm{~cd}$ & $6.08 \mathrm{~d}$ & $18.76 \mathrm{de}$ & $4.59 \mathrm{~b}-\mathrm{d}$ & $258.00 \mathrm{c}$ & $210.00 \mathrm{~d}$ & $180.00 \mathrm{~d}$ & $1.71 \mathrm{~cd}$ & $7.75 \mathrm{e}$ \\
\hline & Amino & $19.25 \mathrm{~cd}$ & $4.71 \mathrm{a}-\mathrm{c}$ & $235.00 \mathrm{~cd}$ & $196.50 \mathrm{~cd}$ & $165.00 \mathrm{~cd}$ & $1.79 \mathrm{~cd}$ & $7.22 \mathrm{c}$ & $19.25 \mathrm{~cd}$ & $4.92 \mathrm{a}-\mathrm{d}$ & $260.00 \mathrm{c}$ & $221.50 \mathrm{~cd}$ & $185.00 \mathrm{~cd}$ & $1.795 \mathrm{~d}$ & $8.63 \mathrm{c}-$ \\
\hline & Humic & $19.50 \mathrm{~cd}$ & $4.66 \mathrm{bc}$ & $233.00 \mathrm{~d}$ & $187.50 \mathrm{~d}$ & $163.00 \mathrm{~d}$ & $1.72 \mathrm{~cd}$ & $7.00 \mathrm{c}$ & $19.25 \mathrm{~cd}$ & $4.89 \mathrm{a}-\mathrm{d}$ & $258.00 \mathrm{c}$ & $212.50 \mathrm{~d}$ & $183.00 \mathrm{~d}$ & $1.72 \mathrm{~cd}$ & $8.08 \mathrm{de}$ \\
\hline \multirow{3}{*}{ Cattle } & Control & $20.16 \mathrm{~b}-\mathrm{c}$ & $4.80 \mathrm{a}-\mathrm{c}$ & $241.50 \mathrm{~b}-\mathrm{d}$ & $200.00 \mathrm{~b}-\mathrm{d}$ & $171.00 \mathrm{~b}-\mathrm{d}$ & $1.85 \mathrm{bc}$ & $7.63 \mathrm{bc}$ & $20.01 b-d$ & $5.07 \mathrm{a}-\mathrm{c}$ & $266.50 \mathrm{c}$ & $225.00 \mathrm{~cd}$ & $191.00 b-d$ & $1.85 \mathrm{bc}$ & $8.20 \mathrm{de}$ \\
\hline & Amino & $20.40 \mathrm{bc}$ & $4.94 \mathrm{a}-\mathrm{c}$ & $249.50 \mathrm{bc}$ & $205.00 \mathrm{bc}$ & $175.00 \mathrm{~b}-\mathrm{d}$ & $1.88 \mathrm{bc}$ & $8.26 \mathrm{~b}$ & $20.28 \mathrm{bc}$ & $5.24 \mathrm{ab}$ & $274.50 \mathrm{cb}$ & $230.00 \mathrm{~cd}$ & $195.00 \mathrm{~b}-\mathrm{d}$ & $1.88 \mathrm{bc}$ & $9.26 \mathrm{c}$ \\
\hline & Humic & $20.40 \mathrm{bc}$ & $4.82 \mathrm{a}-\mathrm{c}$ & $244.00 b-d$ & $200.00 \mathrm{bd}$ & $172.50 \mathrm{~b}-\mathrm{d}$ & $1.87 \mathrm{bc}$ & $7.78 \mathrm{bc}$ & $20.10 \mathrm{~b}-\mathrm{d}$ & $5.13 \mathrm{ab}$ & $269.00 \mathrm{bc}$ & $227.50 \mathrm{~cd}$ & $192.50 b-d$ & $1.87 \mathrm{bc}$ & $8.78 \mathrm{cc}$ \\
\hline \multirow[b]{2}{*}{ Chicken } & Control & $20.55 \mathrm{bc}$ & $5.03 \mathrm{ab}$ & $251.50 \mathrm{~b}$ & $205.00 \mathrm{bc}$ & $182.50 \mathrm{bc}$ & $1.93 \mathrm{bc}$ & $9.59 \mathrm{~b}$ & $20.35 \mathrm{bc}$ & 5.31a & $277.50 \mathrm{bc}$ & $237.50 \mathrm{bc}$ & $202.50 \mathrm{bc}$ & $1.93 \mathrm{bc}$ & $9.59 \mathrm{c}$ \\
\hline & Amino & $21.39 \mathrm{ab}$ & $5.11 \mathrm{a}$ & $300.00 \mathrm{a}$ & $227.50 \mathrm{a}$ & $200.00 \mathrm{a}$ & $2.25 \mathrm{a}$ & $10.96 \mathrm{a}$ & $22.12 \mathrm{a}$ & $5.34 \mathrm{a}$ & $313.00 \mathrm{a}$ & $265.00 \mathrm{a}$ & $235.00 \mathrm{a}$ & $2.25 \mathrm{a}$ & $11.70 \mathrm{a}$ \\
\hline
\end{tabular}

Egypt. J. Hort. Vol. 43, No. 2 (2016) 


\section{Kernels quality}

Concerning the results of different fertilizer sources and growth stimulants and their interactions on kernels quality of sweet corn are listed in Table 5. The results revealed that using chicken manure fertilizer, significantly, increased the five characters, kernels dry matter, reducing sugars, non-reducing sugars, total sugars, and T.S.S. compared to the other treatments, during the two summer seasons. Also, using cattle manure fertilizer did not reflect any significant differences from the application by chicken manure in the case of non-reducing sugars character. The same results was given by Moursy et al., 2007, who found that addition organic manure reflected significant effect for T.S.S. of onion bulb. Significant differences were detected for the starch content as a result of applying the fertilizer sources and the best concentration was given by the application of chicken manure, in the two growing seasons.

TABLE 5. Effect of fertilizer sources, growth stimulants and their interactions on percentages of dry matter, reducing sugars, non-reducing sugars, total sugars, starch and TSS of sweet corn kernels, during the seasons of 2014 and 2015.

\begin{tabular}{|c|c|c|c|c|c|c|c|c|c|c|c|c|c|}
\hline \multicolumn{2}{|c|}{$\begin{array}{l}\text { Seasons } \\
\end{array}$} & \multicolumn{6}{|c|}{2014} & \multicolumn{6}{|c|}{2015} \\
\hline \multicolumn{2}{|c|}{ Treatments } & \begin{tabular}{|c|}
$\begin{array}{c}\text { Kernels } \\
\text { dry } \\
\text { matter } \\
(\%)\end{array}$ \\
\end{tabular} & $\begin{array}{l}\text { Reducin } \\
\text { g sugars } \\
(\%)\end{array}$ & \begin{tabular}{|c|} 
No- \\
Reducing \\
sugars \\
$(\%)$
\end{tabular} & $\begin{array}{c}\text { Total } \\
\text { sugars } \\
(\%)\end{array}$ & $\begin{array}{c}\text { Starch } \\
(\%)\end{array}$ & $\begin{array}{l}\text { T.S.S. } \\
(\%)\end{array}$ & \begin{tabular}{|c|}
$\begin{array}{c}\text { Kernels } \\
\text { dry } \\
\text { matter } \\
(\%)\end{array}$ \\
\end{tabular} & $\begin{array}{l}\text { Reducin } \\
\text { g sugars } \\
(\%)\end{array}$ & $\begin{array}{c}\text { No- } \\
\text { Reducing } \\
\text { sugars } \\
(\%)\end{array}$ & $\begin{array}{c}\text { Total } \\
\text { sugars } \\
(\%)\end{array}$ & $\begin{array}{c}\text { Starch } \\
(\%)\end{array}$ & $\begin{array}{l}\text { T.S.S. } \\
(\%)\end{array}$ \\
\hline \multicolumn{14}{|c|}{ Fertilizer sources } \\
\hline \multicolumn{2}{|c|}{ Control } & $35.431 \mathrm{c}$ & $5.510 \mathrm{~d}$ & $628 \mathrm{~b}$ & $38 \mathrm{~d}$ & $5.856 \mathrm{a}$ & $11.816 \mathrm{c}$ & $34.630 \mathrm{~d}$ & $5.877 \mathrm{~d}$ & 0. b761 b & 63 & 3 & $\mathrm{~d}$ \\
\hline \multicolumn{2}{|c|}{ Mineral fertilizer } & $44.019 \mathrm{~b}$ & $6.552 \mathrm{c}$ & $0.749 \mathrm{ab}$ & $7.302 \mathrm{c}$ & $4.593 \mathrm{~b}$ & $12.541 \mathrm{~b}$ & $42.780 \mathrm{c}$ & $6.886 \mathrm{c}$ & $0.911 \mathrm{~b}$ & $7.797 \mathrm{c}$ & $4.965 \mathrm{~b}$ & $12.493 \mathrm{c}$ \\
\hline \multirow{2}{*}{\multicolumn{2}{|c|}{$\begin{array}{c}\text { Cattle manure } \\
\text { Chicken manure }\end{array}$}} & $44.293 \mathrm{~b}$ & $8.049 \mathrm{~b}$ & $0.755 \mathrm{ab}$ & $8.804 \mathrm{~b}$ & $3.546 \mathrm{c}$ & $13.641 \mathrm{a}$ & $45.460 \mathrm{~b}$ & $7.848 \mathrm{~b}$ & $1.203 \mathrm{a}$ & $9.051 \mathrm{~b}$ & $4.018 \mathrm{c}$ & $13.594 \mathrm{~b}$ \\
\hline & & $48.193 \mathrm{a}$ & $9.372 \mathrm{a}$ & $0.916 \mathrm{a}$ & $10.288 \mathrm{a}$ & $2.556 \mathrm{~d}$ & $13.985 \mathrm{a}$ & $48.320 \mathrm{a}$ & $9.672 \mathrm{a}$ & 1.1 & $10.853 \mathrm{a}$ & 3.1 & 14.0 \\
\hline \multicolumn{14}{|c|}{ Growth stimulants } \\
\hline \multicolumn{2}{|c|}{ Control } & $40.984 \mathrm{~b}$ & $6.973 \mathrm{c}$ & $0.709 \mathrm{~b}$ & $7.682 \mathrm{c}$ & $4.511 \mathrm{a}$ & $12.662 \mathrm{c}$ & $39.990 \mathrm{c}$ & $7.053 \mathrm{c}$ & $0.935 \mathrm{a}$ & $7.988 \mathrm{c}$ & $4.886 \mathrm{a}$ & $12.595 \mathrm{c}$ \\
\hline \multirow{2}{*}{\multicolumn{2}{|c|}{ Amino acid }} & $44.041 \mathrm{a}$ & $7.807 \mathrm{a}$ & $0.833 \mathrm{a}$ & $8.641 \mathrm{a}$ & $3.755 \mathrm{c}$ & $13.387 \mathrm{a}$ & $45.070 \mathrm{a}$ & $8.157 \mathrm{a}$ & $0.958 \mathrm{a}$ & $9.115 \mathrm{a}$ & $4.278 \mathrm{c}$ & $13.363 \mathrm{a}$ \\
\hline & & $43.927 \mathrm{a}$ & $7.331 \mathrm{~b}$ & $0.744 \mathrm{ab}$ & $8.076 \mathrm{~b}$ & $4.148 \mathrm{~b}$ & $12.937 \mathrm{~b}$ & $43.330 \mathrm{~b}$ & $7.501 \mathrm{~b}$ & $1.150 \mathrm{a}$ & $8.651 \mathrm{~b}$ & $4.591 \mathrm{~b}$ & $12.951 \mathrm{~b}$ \\
\hline \multicolumn{14}{|c|}{ Fertilizer sources x Growth stimulants } \\
\hline \multirow{3}{*}{ Control } & Control & $29.175 \mathrm{~h}$ & $5.119 \mathrm{~h}$ & $0.519 \mathrm{e}$ & $5.638 \mathrm{k}$ & $6.265 \mathrm{a}$ & $11.20 \mathrm{e}$ & $26.760 \mathrm{i}$ & $5.419 \mathrm{j}$ & $0.719 \mathrm{~cd}$ & $6.138 \mathrm{i}$ & $6.475 \mathrm{a}$ & $11.257 \mathrm{~d}$ \\
\hline & Amino & $40.105 \mathrm{f}$ & $5.886 \mathrm{f}$ & $0.667 \mathrm{c}-\mathrm{e}$ & $6.553 \mathrm{i}$ & $5.430 \mathrm{c}$ & $12.30 \mathrm{~cd}$ & $40.210 \mathrm{~g}$ & $6.386 \mathrm{~h}$ & $0.665 \mathrm{~d}$ & $7.051 \mathrm{gh}$ & $5.695 \mathrm{c}$ & $12.204 \mathrm{bc}$ \\
\hline & Humic & $37.015 \mathrm{~g}$ & $5.526 \mathrm{~g}$ & $0.619 \mathrm{de}$ & $6.225 \mathrm{j}$ & $5.875 \mathrm{~b}$ & $11.95 \mathrm{~d}$ & $37.010 \mathrm{~h}$ & $5.826 \mathrm{i}$ & $0.899 \mathrm{a}-\mathrm{d}$ & $6.725 \mathrm{~h}$ & $6.155 \mathrm{~b}$ & $11.758 \mathrm{~cd}$ \\
\hline \multirow{3}{*}{ Mineral } & Control & $41.865 \mathrm{ef}$ & $6.118 \mathrm{f}$ & $0.699 \mathrm{c}-\mathrm{e}$ & $6.896 \mathrm{~h}$ & $4.905 \mathrm{~d}$ & $12.30 \mathrm{~cd}$ & $41.860 \mathrm{fg}$ & $6.518 \mathrm{~h}$ & $0.858 \mathrm{~b}-\mathrm{d}$ & $7.376 \mathrm{~g}$ & $4.990 \mathrm{e}$ & $12.253 \mathrm{bc}$ \\
\hline & Amino & $43.33 \mathrm{de}$ & $6.868 \mathrm{e}$ & 0.738 b-d & $7.601 \mathrm{~g}$ & $4.280 \mathrm{f}$ & $12.60 \mathrm{bc}$ & 43.680df & $7.168 \mathrm{fg}$ & $0.933 \mathrm{a}-\mathrm{d}$ & $8.101 \mathrm{f}$ & & $12.752 \mathrm{~b}$ \\
\hline & Humic & $43.155 \mathrm{de}$ & $6.672 \mathrm{e}$ & $0.733 \mathrm{~b}-\mathrm{e}$ & $7.41 \mathrm{~g}$ & $4.595 \mathrm{e}$ & $12.475 \mathrm{~b}-\mathrm{d}$ & $42.800 \mathrm{ef}$ & $6.972 \mathrm{~g}$ & $0.943 \mathrm{a}-\mathrm{d}$ & $7.915 \mathrm{f}$ & $5.300 \mathrm{~d}$ & $12.471 \mathrm{~b}$ \\
\hline \multirow{3}{*}{ Cattle } & Control & $44.545 \mathrm{c}-\mathrm{e}$ & $7.933 \mathrm{~d}$ & $0.765 \mathrm{~b}-\mathrm{d}$ & $8.552 \mathrm{f}$ & $3.890 \mathrm{~g}$ & $12.850 \mathrm{~b}$ & $44.540 \mathrm{c}-\mathrm{e}$ & $7.344 \mathrm{f}$ & $1.199 \mathrm{a}-\mathrm{c}$ & $8.543 \mathrm{e}$ & $4.330 \mathrm{~g}$ & $12.806 \mathrm{~b}$ \\
\hline & Amino & $46.395 \mathrm{bc}$ & $8.19 \mathrm{~d}$ & $0.778 \mathrm{~b}-\mathrm{d}$ & $9.061 \mathrm{~d}$ & $3.110 \mathrm{i}$ & $14.000 \mathrm{a}$ & $46.210 \mathrm{~b}-\mathrm{d}$ & $8.490 \mathrm{~d}$ & $1.071 \mathrm{a}-\mathrm{d}$ & $9.561 \mathrm{c}$ & $3.875 \mathrm{i}$ & $12.974 \mathrm{a}$ \\
\hline & Humic & $45.15 \mathrm{~b}-\mathrm{d}$ & $8.024 \mathrm{~d}$ & 0.775 b-d & $8.799 \mathrm{e}$ & $3.640 \mathrm{~h}$ & $13.800 \mathrm{a}$ & $45.620 \mathrm{~b}-\mathrm{d}$ & $7.711 \mathrm{e}$ & $1.341 \mathrm{ab}$ & $9.052 \mathrm{~d}$ & $4.055 \mathrm{~h}$ & $13.692 \mathrm{a}$ \\
\hline \multirow{3}{*}{ Chicken } & Control & $46.505 \mathrm{bc}$ & $8.633 \mathrm{c}$ & 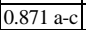 & $9.398 \mathrm{c}$ & \begin{tabular}{|l|}
$2.985 \mathrm{i}$ \\
\end{tabular} & $14.075 \mathrm{a}$ & $46.890 \mathrm{bc}$ & $8.933 \mathrm{c}$ & \begin{tabular}{|c|}
$0.965 \mathrm{a}-\mathrm{d}$ \\
\end{tabular} & $9.898 \mathrm{c}$ & $3.440 \mathrm{j}$ & $14.061 \mathrm{a}$ \\
\hline & Amino & $50.170 \mathrm{a}$ & $10.287 \mathrm{a}$ & $1.062 \mathrm{a}$ & $11.349 \mathrm{a}$ & $2.200 \mathrm{k}$ & $14.250 \mathrm{a}$ & $50.170 \mathrm{a}$ & $10.587 \mathrm{a}$ & $1.162 \mathrm{a}-\mathrm{d}$ & $11.749 \mathrm{a}$ & $2.945 \mathrm{l}$ & $14.364 \mathrm{a}$ \\
\hline & Humic & $47.905 \mathrm{ab}$ & \begin{tabular}{|l|l|}
$9.196 \mathrm{~b}$ \\
\end{tabular} & $0.921 \mathrm{ab}$ & $10.117 \mathrm{~b}$ & $2.485 \mathrm{j}$ & $14.150 \mathrm{a}$ & $47.900 \mathrm{ab}$ & $9.496 \mathrm{~b}$ & $1.418 \mathrm{a}$ & $10.914 \mathrm{~b}$ & $3.160 \mathrm{k}$ & $14.133 \mathrm{a}$ \\
\hline
\end{tabular}

Regarding the main effect of foliar spray by amino - and humic acids on kernels quality of sweet corn, the results pointed out that the application of amino acid foliar spray were significantly associated with corresponding increases in all mean values of the studied kernels quality components, except starch character. Moreover, humic acid foliar spray gave the same results for the two characters kernels dry matter and non-reducing sugars with insignificant differences with the application of amino acid treatment, in the first season and non-reducing sugars, in the second season. Application of amino acid foliar spray gave, significantly, the lowest (desirable) mean value, followed by humic 
acid and control treatments, in the two growing seasons. Fruit dry matter and TSS content of tomato increased significantly with foliar application of humic acid, Kazemi (2014).

Respecting the interaction effect between the two studied factors, fertilizer sources and growth stimulants, in both growing seasons, resulted in steady corresponding increments, with significant differences in the most studied kernels quality components. Application of chicken manure fertilizer with amino acid gave significantly the highest mean values of the most studied characters of kernels quality.

\section{Chemical composition of leaves}

Data presented in Table 5 showed the effects of all studied factors on nutrient contents, N, P and $\mathrm{K}$ of sweet corn leaves. The results reflected that the concentration of phosphorus in sweet corn leaves did not reflect any significant increases as a result of applying the sources of fertilizers, compared with control treatment. Nevertheless, significant differences were detected for both nitrogen and potassium elements. The highest mean values of $\mathrm{N}$ and $\mathrm{K}$ content was given by the application of organic fertilizer (chicken or cattle manures), followed by NPK mineral fertilizer with significant differences. Hu and Barker (2004) on tomato and Mohammed (2011) on pepper mentioned that the organic fertilizer application led to higher concentration and total accumulation of NPK in leaves.

As for the effects of foliar application of the used amino - and humic acids, generally, the results indicated clearly that the concentrations of phosphorus in sweet corn leaves did not reflect any significant increases as a result of applying the two sources of growth stimulants (amino - or humic acids), compared to the control treatment, in the two growing seasons. While, significant differences were detected for the nitrogen and potassium leaves content compared to the control, in the two seasons, with one exception for potassium leaves content in the second season, where, foliar spraying by amino - or humic acids did not recorded any significant differences with control treatment. Similar results were obtained by Kazemi (2014), who reported that sprayed by humic acid resulted in a significant increase in $\mathrm{N}$ and $\mathrm{K}$ content of tomato leaves. Also, Khaled and Fawy (2011) stated that foliar application of humic acid had a significant effect on $\mathrm{P}$ and $\mathrm{K}$ uptake in corn.

The comparisons among the various treatment combinations which reflecting the interaction effect between the two studied main factors, Table 5, showed some positive and significant interactions effects on the performance of the mineral contents ( $\mathrm{N}$ and $\mathrm{K}$ ) of sweet corn leaves, while, leaves $\mathrm{P}$ content means did not affect by the interaction applications. Generally, it was noticed that application by chicken manure fertilizer, combined with amino acid or humic acid foliar spray, lead to marked increased on mean values of mineral contents of ( $\mathrm{N}$ and $\mathrm{K}$ ) of leaves.

Egypt. J. Hort. Vol. 43, No. 2 (2016) 
TABLE 6. Effect of fertilizer sources, growth stimulants and their interactions on percentages of nitrogen, phosphorus and potassium contents of sweet corn leaves, during the seasons of 2014 and 2015.

\begin{tabular}{|c|c|c|c|c|c|c|c|}
\hline \multirow{2}{*}{\multicolumn{2}{|c|}{\begin{tabular}{|c|} 
Seasons \\
Characters \\
Treatments
\end{tabular}}} & \multicolumn{3}{|c|}{2014} & \multicolumn{3}{|c|}{2015} \\
\hline & & $\mathbf{N}(\%)$ & $P(\%)$ & $\mathbf{K}(\%)$ & $\mathbf{N}(\%)$ & $\mathbf{P}(\%)$ & $\mathbf{K}(\%)$ \\
\hline \multicolumn{8}{|c|}{ Fertilizer sources } \\
\hline \multicolumn{2}{|c|}{ Control } & $1.00 \mathrm{~d}$ & $0.12 \mathrm{a}$ & $1.02 \mathrm{~d}$ & $1.10 \mathrm{c}$ & $0.25 \mathrm{a}$ & $0.92 \mathrm{c}$ \\
\hline \multicolumn{2}{|c|}{ Mineral fertilizer } & $1.52 \mathrm{c}$ & $0.16 \mathrm{a}$ & $1.39 \mathrm{c}$ & $1.86 \mathrm{~b}$ & $0.30 \mathrm{a}$ & $1.56 \mathrm{~b}$ \\
\hline \multicolumn{2}{|c|}{ Cattle manure } & $2.37 \mathrm{~b}$ & $0.23 \mathrm{a}$ & $1.75 \mathrm{~b}$ & $2.13 \mathrm{a}$ & $0.31 \mathrm{a}$ & $2.41 \mathrm{a}$ \\
\hline \multicolumn{2}{|c|}{ Chicken manure } & $2.53 \mathrm{a}$ & $0.32 \mathrm{a}$ & $2.35 \mathrm{a}$ & $2.41 \mathrm{a}$ & $0.47 \mathrm{a}$ & $2.57 \mathrm{a}$ \\
\hline \multicolumn{8}{|c|}{ Growth stimulants } \\
\hline \multicolumn{2}{|c|}{ Control } & $1.76 \mathrm{c}$ & $0.18 \mathrm{a}$ & $1.45 \mathrm{c}$ & $2.01 \mathrm{~b}$ & $0.27 \mathrm{a}$ & $1.77 \mathrm{a}$ \\
\hline \multicolumn{2}{|c|}{ Amino acid } & $2.14 \mathrm{a}$ & $0.22 \mathrm{a}$ & $1.81 \mathrm{a}$ & $2.24 \mathrm{a}$ & $0.27 \mathrm{a}$ & $2.01 \mathrm{a}$ \\
\hline \multicolumn{2}{|c|}{ Humic acid } & $1.96 \mathrm{~b}$ & $0.21 \mathrm{a}$ & $1.63 \mathrm{~b}$ & $2.21 \mathrm{a}$ & $0.26 \mathrm{a}$ & $1.81 \mathrm{a}$ \\
\hline \multicolumn{8}{|c|}{ Fertilizer sources x Growth stimulants } \\
\hline \multirow{3}{*}{ Control } & Control & 0.811 & $0.10 \mathrm{a}$ & $0.92 \mathrm{i}$ & $0.85 \mathrm{~g}$ & $0.21 \mathrm{a}$ & $0.73 \mathrm{~g}$ \\
\hline & Amino & $1.17 \mathrm{j}$ & $0.11 \mathrm{a}$ & $1.17 \mathrm{~h}$ & $1.53 \mathrm{f}$ & $0.25 \mathrm{a}$ & $1.12 \mathrm{eg}$ \\
\hline & Humic & $1.03 \mathrm{k}$ & $0.12 \mathrm{a}$ & $0.99 \mathrm{i}$ & $1.25 \mathrm{f}$ & $0.23 \mathrm{a}$ & $0.92 \mathrm{fg}$ \\
\hline \multirow{3}{*}{ Mineral } & Control & $1.34 \mathrm{i}$ & $0.13 \mathrm{a}$ & $1.21 \mathrm{~h}$ & $1.61 \mathrm{ef}$ & $0.27 \mathrm{a}$ & $1.36 \mathrm{~d}-\mathrm{f}$ \\
\hline & Amino & $1.71 \mathrm{~g}$ & $0.16 \mathrm{a}$ & $1.50 \mathrm{fg}$ & $1.99 \mathrm{c}-\mathrm{e}$ & $0.28 \mathrm{a}$ & $1.83 \mathrm{~cd}$ \\
\hline & Humic & $1.5 \mathrm{~h}$ & $0.18 \mathrm{a}$ & $1.46 \mathrm{~g}$ & $1.97 \mathrm{de}$ & $0.28 \mathrm{a}$ & $1.48 \mathrm{de}$ \\
\hline \multirow{3}{*}{ Cattle } & Control & $2.11 \mathrm{f}$ & $0.21 \mathrm{a}$ & 1.64 ef & $2.12 \mathrm{~cd}$ & $0.29 \mathrm{a}$ & $2.12 b c$ \\
\hline & Amino & $2.61 \mathrm{~d}$ & $0.24 \mathrm{a}$ & $1.86 \mathrm{~d}$ & $2.37 \mathrm{c}$ & $0.32 \mathrm{a}$ & $2.56 \mathrm{ab}$ \\
\hline & Humic & $2.39 \mathrm{e}$ & $0.26 \mathrm{a}$ & $1.75 \mathrm{de}$ & $2.17 \mathrm{~cd}$ & $0.29 \mathrm{a}$ & $2.25 \mathrm{bc}$ \\
\hline \multirow{3}{*}{ Chicken } & Control & $2.78 \mathrm{c}$ & $0.28 \mathrm{a}$ & $2.04 \mathrm{c}$ & $2.93 \mathrm{~b}$ & $0.34 \mathrm{a}$ & $2.58 \mathrm{ab}$ \\
\hline & Amino & $3.1 \mathrm{a}$ & $0.34 \mathrm{a}$ & $2.68 \mathrm{a}$ & $3.57 \mathrm{a}$ & $0.43 \mathrm{a}$ & $2.85 \mathrm{a}$ \\
\hline & Humic & $2.92 \mathrm{~b}$ & $0.34 \mathrm{a}$ & $2.34 \mathrm{~b}$ & $3.45 \mathrm{ab}$ & $0.35 \mathrm{a}$ & $2.58 \mathrm{ab}$ \\
\hline
\end{tabular}

Conclusion and Recommendation

From this experiment, application of chicken manure in combination with amino - or humic acids foliar spray showed better performance than the other treatments. So, it can be concluded that use of chicken manure can meet the crop nutrient demand throughout the growth stages or increasing yield and quality of corn through improvement the soil physical, chemical and biological properties. Moreover, amino - and humic acids have directly or indirectly influence the physiological activities of the plant.

\section{References}

Abou El-Magd, M.M., El-Bassiony, M. and Fawzy, Z.F. (2006) Effect of organic manure with or without chemical fertilizers on growth, yield and quality of some varieties of broccoli plants, J. Appl. Sci. Res., 2 (10), 791-798.

Abo Sedera, F.A., Abd El-Latief, A.A. Bader, L.A. and Rezk, S.M. (2010) Effect of NPK mineral fertilizer levels and foliar application with humic and amino acids on yield and quality of strawberry. Egypt. J. Appl. Sci., 25, 154-169.

Adediran, J.A., Taiwo, L.B., Akande, M.O., Sobula R.A. and Idowu, O.J. (2004) Application of organic and inorganic fertilizer for sustainable maize and cowpea yields in Nigeria. J. Pl. Nut., 27 (7), 1163-1170.

Egypt. J. Hort. Vol. 43, No. 2 (2016) 
Amanolahi-Baharvand, Z., Zahedi H. and Rafiee, M. (2014) Effect of Vermicompost and chemical fertilizers on growth parameters of three corn cultivars. J. Appl. Sci. Agric., 9 (9), 22-26.

A.O.A.C. (1990) "Association Official Agriculture Chemists", $13^{\text {th }}$ ed., Washington, D. C., USA.

Ayoola, S.R. and Makinde, E.A. (2009) Maize growth, yield and soil nutrient change with N-enriched organic fertilizers. African J. Food Agric. Nut. \& Develop., 9 (1), 580-592.

Azimi, M.S., Daneshian, J., Sayfzadeh, S. and Zare, S. (2013) Evaluation of amino acid and salicylic acid application on yield and growth of wheat under water deficit. Inter. J. Agric. \& Crop Sci., 5 (8), 816-819.

Boateng, S.A., Zickermann, J. and Kornahrens, M. (2006) Poultry manure effect on growth and yield of maize. West Africa J. Appl. Eco., 9, 12-18.

Cenellar, L.P., Olivarer, F.L., Okookova-FA, A.L. and Facanha, A.R. (2002) Humic acids isolated from earth warm compost enhance root elongation, lateral root emergence and plasma membrane H-ATP base activity in maize roots. Pl. Phys., 130, 1951-1957.

Chapman, H.D. and Pratt, F. (1961) Methods of soil analyses. Part2 A.S.S. madisonwiscoas in course puble. By the author, Dep. of Soils, Univ. of Wise., Madison 6, Wishensin, U.S.A.

Chen, Y., Clapp, C.E. and Magen, H. (2004) Mechanisms of plant growth stimulation by humic substances: The role of organic-iron complexes. Soils Sci. \& Pl. Nut., 50, 1089-1095.

Co-State Software (2004) User's manual version. Cohort Tusson, Arizona, USA.

Dikinya, O. and Mufwanzala, N. (2010) Chicken manure-enhanced soil fertility and productivity: Effect of application rates. J. Soil Sci. \& Environ. Manage., 1(3): 46-54.

Dinesh, R., Srinivasan, V., Hamza, S. and Manjusha, A. (2010) Short-term incorporation of organic manures and biofertilizers influences biochemical and microbial characteristics of soil under an annual crop (Turmeric (Curcuma longa L.). Bioresour Technol., 101, 4697-4702.

Doehlert, D.C., Kuo, T.M., Juvik, J.A. Beers, E.P. and Duke, S.H. (1993) Characteristics of carbohydrate metabolism in sweet corn sugary-1 endosperms. $J$. Amer. Soc. Hort. Sci., 118 (5), 661-666.

Ebrahimi, M., Roozbahani, A. and Baghi, M. (2014) Effect of potash fertilizer and amino acids on yield components and yield of maize (Zea mays L.). Crop Res., 48 $(1,2 \& 3), 15-21$.

Egypt. J. Hort. Vol. 43, No. 2 (2016) 
Efthimiadou, A, Bilalis, D. and Karkanis, A. (2009) Effects of cultural system (organic and conventional) on growth, photosynthesis and yield components of sweet corn (Zea mays L.) under semi-arid environment. Notula Botanicae Hort. Agrobotanicicluj-Napoca. 37 (2), 438-439.

Evenhuis, B. and Dewaord, P.W. (1980) Principles and practices in plant analysis. F.A.O. Soil Bull., 38 (1), 152-163.

Faten, S.A., Shaheen, A.M., Ahmed, A.A. and Mahmoud, A.R. (2010) Effect of foliar application of amino acids as antioxidants on growth, yield and characteristics of squash. Res. J. Agric. and Biol. Sci., 6, 583-588.

Geisseler, D., Horwath, W.R. Joergensen, R.G. and Ludwig, B. (2010) Pathway of nitrogen utilization by soil microorganisms- a review. Soil Biol. and Biochemist. 42, (2058-2067).

Ghosh, B.C., Bera, N., Das, D. and Swain, D.K. (2013) Effect of varying soil and vermicompost mixtures on growth meadia and yield quality of sweet corn. Int. Confer. Food \& Agri. Sci., 55 (8), 38-42.

Gudugi, I.A., Isah M.K. and Giragi, A.N. (2012) Effect of different rates of poultry manure on the growth and yield of sweet corn (Zea mays var. saccharata). Indian J. Res., 3 (2), 13-16.

Hu, Y.F. and Barker, A.V. (2004) Effect of composts and their combinations with other materials on nutrients accumulation in tomato leaves. Comm. Soil Sci. \& Pl. Anal. 35 (19120), 2809-2823.

Inselsbachr, E., Hinko-Najera, N., Stange, F.C., Gorfer, M., Schuller, E., Ripka, K., Zechmeister-Boltenstern, S., Hood-Novotny, R., Strauss, J. and Wanek, W. (2010) Short-term competition between crop plants and soil microbes for inorganic $\mathrm{N}$ fertilizer. Soil Bio. \& Biochemist., 42, 360-372.

Jasim, A.H., Helmee, H. and Abdullah, K.N. (2014) The effect of type and level of organic fertilizer and foliar of humic acid on corn (Zea mays L.) yield and its components. Int. Conf. Adv. Environ. Agric. \& Medic. Sci., November (16-17), 28-33.

Kandil, A.A., Sharief, A.E. and Fathalla, F.H. (2013) Onion yield as affected by foliar application with amino and humic acids under nitrogen fertilizer levels. J. Crop Prod., 2 (2), 2305-2627.

Kazemi, M. (2014) Effect of foliar application of humic acid and calcium chloride on tomato growth. Bull. Environ. Pharm. \& Life Sci., 3 (3), 41-46.

Khaled, H. and Fawy, H.A. (2011) Effect of different levels of humic acids on the nutrient content, plant growth, and soil properties under conditions of salinity. Soil and Water Res., 6 (1), 21-29. 
Lazcano, C., Revilla, P., Malvar, R. and Domingu, J. (2011) Yield and fruit quality of four sweet corn hybrids (Zea mays) under conventional and integrated fertilization with vermicompost. J. Sci. Food Agric., 91(7), 1244-1253.

Lazcano, C., Gomez-Brandon, M. Revilla, P and Dominguez, J. (2012) Short-term effects of organic and inorganic fertilizers on soil microbial community structure and function: Afield study with sweet corn. Biol. Fertil. Soils.

Materechera, S.A. and Salaga, A.M. (2002) Use of partially-decomposed cattle and chicken manure a mended with wood-ash in two South African arable soils with contrasting texture: Effect on nutrient uptake, early growth and dry matter yield of maize. Comm. Soil Sci. \& Pl. Anal., 33, 179-201.

Mohammed, S.M. (2011) Pepper response to organic and biofertilizers under plastic house condition. M.Sc. Thesis. Faculty of Agriculture, Cairo University, Cairo, Egypt.

Moursy, M.El., Khalifa, H.E., Attia, M.M., Saged M.A. and Osman, A.M. (2007) Effect of organic and nitrogen fertilizers and plant densities on onion production in sandy soil under drip irrigation system. Alex. J. Agri. Res., 52 (1), 103-108.

Murmur, K., Swain, D.K. and Ghosh, B.C. (2013) Comparative assessment of conventional and organic nutrient management on crop growth and yield and soil fertility in tomato-sweet corn production system. Austral. J. Crop Sci., 7 (11), $1617-1626$.

Noble, R. and Coventry, E. (2005) Suppression of soil-borne plant diseases with composts: A review. Bio-control Sci. \& Techno., 15, 2-20.

Oad, F.C, Burrio, U.A. and Agha, S.K. (2004) Effect of organic and inorganic fertilizer application on maize fodder production. Asian. J. Pl. Sci. 3: 375-377. Appl. Sci., 1 (3), 187-192.

Obi, M.E. and Ebo, P.O. (1995) The effects of different application rates of organic and inorganic fertilizers on soil physical properties and maize production in a severely degraded ultisol in southern Nigeria. Bioresourc. Technol., 51 (2), 117-123.

Obi, A.H., Izundu, C. and Lliyasu, A., (2013) Effect of chicken manure on the performance of vegetable mize (Zea mays var. saccharata) varieties under irrigation. Discourse J. Agri. \& Food Sci., 1 (12), 190-195.

Page, A.L., Miller, R.H. and Reeney, D.R., (1982) "Methods of Soil Analysis", part 2. ASA, SSSA, Madison, Wisconsin, USA.

Saeed, M.R., Kheir, A.M. and Al-Sayed, A.A. (2005) Suppressive effect of some amino acids against Meloidogyne incognita on soybean. J. Agri. Sci. Mansoura Univ., 30 (2), 1097- 1103 .

Egypt. J. Hort. Vol. 43, No. 2 (2016) 
Shalaby, T.A. and El-Ramady, H. (2014) Effect of foliar application of bio-stimulants on growth, yield, components, and storability of garlic (Allium sativumL.). Austral. J. Crop Sci., (AJCS). 8 (2), 271-275.

Simon, S.R. and Balabbo, F.P. (2015) Yield performance of sweet corn (Zea mays var. saccharata) using vermicompost as a component of balanced fertilization strategy. Int. J. Chemical, Environment. \& Biological Sci. (IJCEBS)., 3 (3), 224-227.

Steel, R.G.D. and Torrie, F.H. (1980) "Principles and Procedures of Statistics", $2^{\text {nd }}$ ed., McGraw Hill Book Co., New York.

Toth, S. J., Prince, A.L., Wallace, A. and MikKelsen (1948) Rapid quantitative determination of eight mineral elements in plant tissue by a systematic procedure involving use of flame photometer. Soil Sci., 66, 456-466.

(Received 15/9/2016; accepted 29/11/ 2016) 


\section{تأثير الرش بالهيوميك والأحماض الأمينية على الذرة السكرية تحت مصادر مختلفة من التسميد \\ انتصار إبر اهيم مسعود راغب اغلة اغلة \\ قسم الخضر - كلية الزر اعة - جامعة الإسكندرية ـ الإسكندرية - مصر.}

تم تنفيذ تجربتان حقليتان بمزرعة كلية الزر اعة - جامعة الإسكندرية بمنطقة أبيس

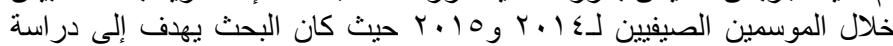

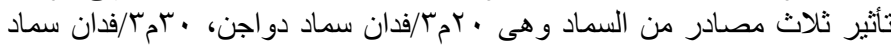

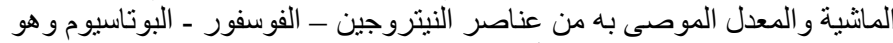

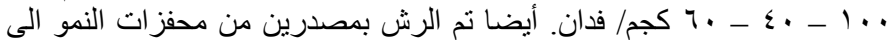

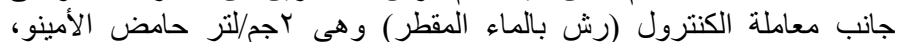
وك.r.o

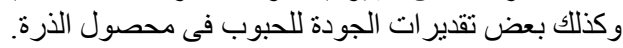

تم تنفيذ تجربتان حقليتان بمزرعة كلية الزراعة - جامعة الإسكندرية بمنطقة

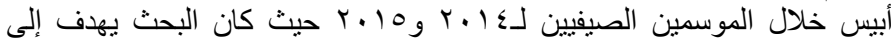

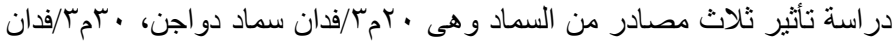

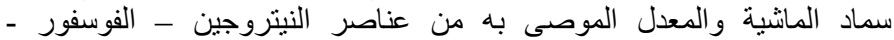

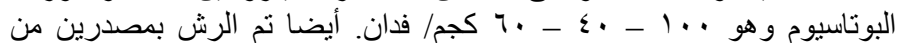

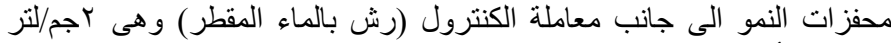

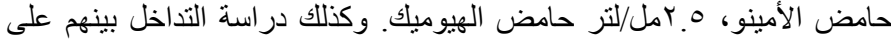

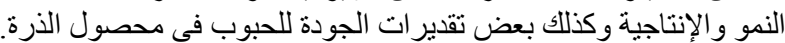

وأظهرت النتائج أن استخدام سماد الدو اجن على الذرة السكرية قد عكس زيادة

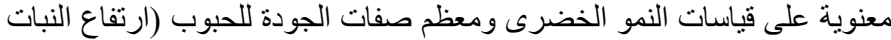

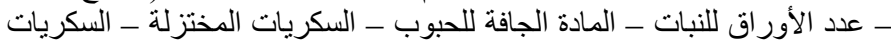

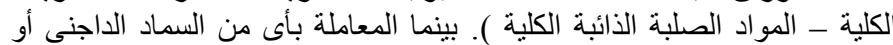

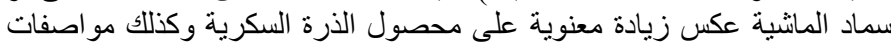

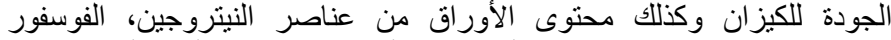

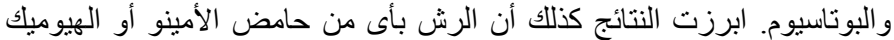

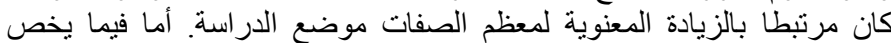

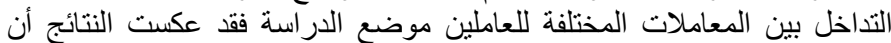

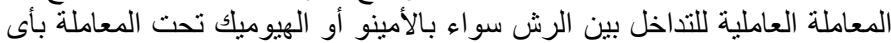

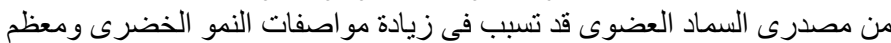

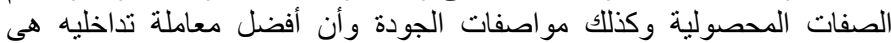

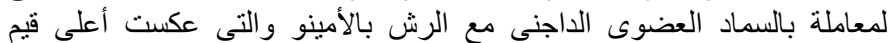
للمتوسطات بالنسبة للصفات المدروسة.

Egypt. J. Hort. Vol. 43, No. 2 (2016) 